\title{
Originalien
}

Notfall Rettungsmed 2021 $\cdot 24: 943-952$ https://doi.org/10.1007/s10049-020-00757-w Online publiziert: 8 . Juli 2020

(c) Der/die Autor(en) 2020

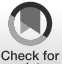

T. Tschaikowsky ${ }^{1,2} \cdot$ A. Becker von Rose ${ }^{3} \cdot$ S. Consalvo ${ }^{4} \cdot$ P. Pflüger ${ }^{4} \cdot$ P. Barthel ${ }^{1}$ C. D. Spinner ${ }^{5} \cdot$ B. Knier $\cdot$ K.-G. Kanz ${ }^{2,4} \cdot$ M. Dommasch ${ }^{1,2}$

${ }^{1}$ Fakultät für Medizin, Klinikum rechts der Isar, Innere Medizin I, Technische Universität München, München, Deutschland

${ }^{2}$ Zentrale interdisziplinäre Notaufnahme, Klinikum rechts der Isar, Technische Universität München, München, Deutschland

${ }^{3}$ Fakultät für Medizin, Klinikum rechts der Isar, Innere Medizin III, Technische Universität München, München, Deutschland

${ }^{4}$ Fakultät für Medizin, Klinikum rechts der Isar, Unfallchirurgie, Technische Universität München, München Deutschland

${ }^{5}$ Fakultät für Medizin, Klinikum rechts der Isar, Innere Medizin II, Technische Universität München, München, Deutschland

${ }^{6}$ Fakultät für Medizin, Klinikum rechts der Isar, Neurologie, Technische Universität München, München, Deutschland

\section{Patientenzahlen im Rahmen der COVID-19-Pandemie in einer zentralen Notaufnahme}

durch Kontaktbeschränkungen ersetzt wurden [1, 4, 6, 11]. Im Zusammenhang mit dem Katastrophenfall haben das Bayerische Staatsministerium des Innern, für Sport und Integration sowie das Bayerischen Staatsministeriums für Gesundheit und Pflege am 24.03.2020 eine Allgemeinverfügung erlassen, in der unter anderem die Organisation der Krankenhausbelegung, Neukonzeption der IT-Steuerung und Meldepflichten, Schaffung zusätzlicher Kapazitäten, COVID-19-Koordinierungsgruppen der Krankenhäuser sowie organisatorische Maßnahmen für Krankenhäuser angeordnet wurden [5].

Entsprechend der vorhandenen Pandemie- und Notfallpläne wurden im Raum München schon nach dem Auftreten der ersten COVID-19-Fälle Vorkehrungen im Gesundheitswesen getroffen. Auch aufgrund der Berichterstattung aus Italien, Frankreich und Spanien wurden Krankenhauskapazitäten umfunktioniert und Intensivbetten geschaffen, um einem möglicherweise bevorstehenden großen Ansturm an Patienten gerecht zu werden [2].

Im Rahmen dieser Vorhaltemaßnahmen wurden Betten in Fachbereichen, die hauptsächlich elektive Medizin betrei- ben, reduziert, um andere Bereich, wie z. B. die Notaufnahmen, zu stärken. Mit dem Sonderlagenmodul IVENA (mainis IT-Service GmbH, Offenbach am Main, Deutschland) melden die Kliniken mehrmals täglich den Ist-Stand der Betten an die Sicherheits- und Gesundheitsbehörden.

Die COVID-19-Fälle stiegen täglich weiter an, aber aufgrund der deutlichen und strikten Ausgangsbeschränkungen verlängerte sich auch die Verdopplungszeit der Viruserkrankungen, sodass es erfreulicherweise zu keiner Überlastung der Krankenhäuser in Deutschland gekommen ist.

Alle Vorhaltemaßnahmen, die in den Monaten getroffen wurden, hatten zum Nebeneffekt, dass die medizinische Versorgung auf einen Notbetrieb umgestellt wurde. Die hierdurch entstandenen gesundheitlichen und volkswirtschaftlichen Schäden sind noch nicht absehbar. Diese Arbeit soll das Patientenaufkommen zu Beginn und nach Abflachen der wohl ersten COVID-19-Welle in Deutschland aufzeigen und kann für weitere Pandemieplanungen im Bereich der Notaufnahmen miteinbezogen werden. erlassen, die ab dem 21.03.2020 bis zum 05.05.2020 galten und am 06.05.2020 
Tab. 1 Übersicht über die einzelnen Dringlichkeitsstufen gemäß MTS

\begin{tabular}{|c|c|c|c|}
\hline Triagefarbe & Dringlichkeit & $\begin{array}{l}\text { Zeitpunkt ärztli- } \\
\text { cher Kontakt }\end{array}$ & Beschwerden \\
\hline Blau & Nichtdringend & $<120$ min & Seit Längerem bestehende Beschwerden \\
\hline Grün & Normal & $<90 \min$ & Subakute bis akute Beschwerden ohne Risikozeichen \\
\hline Gelb & Dringend & $<30 \mathrm{~min}$ & $\begin{array}{l}\text { Akute Beschwerden mit Risikozeichen, z. B. Blutungszeichen, mäßige Schmerzen, offene Frak- } \\
\text { turen, Fehlstellungen, neurologisches Defizit oder niedrige periphere Sauerstoffsättigung } \\
\text { (<95\% unter Raumluft) }\end{array}$ \\
\hline Orange & $\begin{array}{l}\text { Sehr drin- } \\
\text { gend }\end{array}$ & $<10 \min$ & $\begin{array}{l}\text { Starke akute Beschwerden, z. B. akute Atemnot, Bluterbrechen, stärkste Schmerzen, insbeson- } \\
\text { dere Thoraxschmerz, veränderter Bewusstseinszustand oder sehr niedrige periphere Sauer- } \\
\text { stoffsättigung ( }<90 \% \text { unter Raumluft) }\end{array}$ \\
\hline Rot & Sofort & $0 \mathrm{~min}$ & $\begin{array}{l}\text { Akute Lebensgefahr, z. B. bei akutem Schockgeschehen, gefährdetem Atemweg, lebensbe- } \\
\text { drohlicher Blutung, anhaltendem Krampfanfall oder Hypoglykämie }\end{array}$ \\
\hline
\end{tabular}

\section{Methoden}

\section{Zentrale Notaufnahme des Klinikums rechts der Isar im Kontext}

Das Klinikum rechts der Isar (RDI) der Technischen Universität München weist gemäß $₫ 136 c$ Absatz 4 des 5. Buches, Sozialgesetzbuch (SGB V) die Voraussetzungen für eine Klinik der „umfassenden Notfallversorgung - Stufe 3" auf [9]. Die zentrale Notaufnahme (ZNA) des Klinikums gehört zu den größten im Rettungsdienstbereich München. Jährlich werden mehr als 40.000 Notfälle in der ZNA behandelt. Sie ist für jegliche Notfälle ausgerüstet und verfügt über einen Schockraumbereich mit Computertomographie, für den ein interdisziplinäres Team aus den Fachbereichen Chirurgie, innere Medizin, Neurologie, Anästhesie und Radiologie vorgehalten wird. Hauptsächlich werden in der ZNA Patienten aus den Fachbereichen Chirurgie, innere Medizin sowie Neurologie behandelt. Zusätzlich stehen am Klinikum Spezialambulanzen der Bereiche Urologie, Gynäkologie, MundKiefer-Gesichtschirurgie, Hals-NasenOhren-Heilkunde und Augenheilkunde zur Verfügung.

Im Zuge der SARS-CoV-2-Pandemie erfuhr die ZNA eine grundlegende Umstrukturierung in einen räumlich getrennten infektiösen und nichtinfektiösen Bereich. Die Patienten werden analog eines an die Gegebenheiten des Klinikums angepassten COVID-19-Algorithmus anhand ihrer Symptome und
Temperatur in die jeweiligen Bereiche getrennt $[15,18]$.

\section{Manchester Triage System}

Bei Vorstellung der Patienten in der ZNA erfolgt nach personeller Identifikation eine medizinische Ersteinschätzung, auch „Triage“ genannt, gemäß einem validierten System, dem Manchester Triage System (MTS; [10]). Anhand von insgesamt 50 Beschwerdebildern und entsprechenden Präsentationsdiagrammen wird die Dringlichkeit des Notfalls festgestellt. Hierdurch wird die maximale Zeit bis zum ersten Arztkontakt definiert und nach einem Ampelprinzip von „blau“ für nichtdringend bis ,rot" für akute Lebensgefahr eingeteilt (• Tab. 1).

\section{Fachbereiche}

Die verschiedenen Fachdisziplinen wurden hinsichtlich einer besseren Übersicht in übergeordnete Fachbereiche zusammengefasst. „Chirurgie" beinhaltet alle chirurgischen Subdisziplinen wie Unfall-, Viszeral-, Thorax-, Gefäß-, Mund-Kiefer-Gesichts-, Neurochirurgie und plastische Chirurgie. Die Fachbereiche innere Medizin und Neurologie werden im Folgenden als „Konservativ“ zusammengefasst. Aufgrund der geringen Fallzahlen werden zur besseren Übersicht alle anderen Fachbereiche, wie Psychiatrie, Urologie, Pädiatrie, Gynäkologie und Geburtshilfe, im Folgenden als „Andere“ aufgeführt.

\section{ERPath}

In der ZNA des Klinikums RDI werden alle patientenbezogenen Daten mittels eines Notaufnahmeninformationssystem (ERPath, eHealth-Tec Innovations $\mathrm{GmbH}$, Berlin, Deutschland) erhoben und gespeichert [8]. Das Produkt ermöglicht eine vereinfachte und an die Notaufnahme angepasste Prozessteuerung inklusive Ersteinschätzung und Dokumentation. Es werden alle personenbezogenen Daten erhoben und gesichert. Das Triageergebnis wird mit Beschwerdebild, dessen Dringlichkeit und genauem Zeitstempel dokumentiert. Dies gilt auch für alle erhobenen Vitalparameter, verabreichte Medikamente und Therapien. Zudem werden alle administrativen Prozesse und klinische Untersuchungsbefunde, wie Anamnese, körperliche Untersuchung, Diagnostik und Konsile, systematisch erfasst.

\section{Datenerhebung}

Im Rahmen einer deskriptiven epidemiologischen Studie wurden in dem Zeitraum vom 01.02.2019 bis zum 30.04.2019 sowie 01.02 .2020 bis 30.04 .2020 die in ERPath erhobenen Daten ausgewertet. Die Daten der im Beobachtungszeitraum erhobenen Notfälle wurden anonymisiert aus dem IT-System ERPath extrahiert und ausgewertet. Eine Signifikanz wird durch $p$-Werte $<0,05$ angezeigt. Es wurde keine Korrektur für mehrfache Tests vorgenommen. Für das Alter wurde der Mann-Whitney-U-Test, für alle anderen Faktoren der $\chi^{2}$-Test angewendet (SPSS Statistics for Windows, 
Notfall Rettungsmed 2021 · 24:943-952 https://doi.org/10.1007/s10049-020-00757-w

(c) Der/die Autor(en) 2020

\section{T. Tschaikowsky · A. Becker von Rose · S. Consalvo · P. Pflüger · P. Barthel · C. D. Spinner · B. Knier · K.-G. Kanz · M. Dommasch}

\section{Patientenzahlen im Rahmen der COVID-19-Pandemie in einer zentralen Notaufnahme}

\section{Zusammenfassung}

Hintergrund. Seit Ende März wurde deutschlandweit das Gesundheitswesen auf einen Notbetrieb umgestellt, um Ressourcen für die sich ausbreitende Coronavirus-disease2019-Pandemie (COVID-19-Pandemie) zu schaffen. Ziel der Arbeit ist es, das Aufkommen von Notfallpatienten zur Zeit der Pandemie zu untersuchen, um Rückschlüsse auf den Einfluss der COVID-19-Pandemie auf das Patientenaufkommen in einer Notaufnahme ziehen zu können.

Material und Methoden. Im Rahmen einer deskriptiven epidemiologischen Studie wurden in dem Zeitraum vom 01.02. bis zum 30.04.2019 sowie vom 01.02. bis zum 30.04.2020 patientenbezogene Daten von insgesamt 19.357 Fällen in der zentralen
Notaufnahme des Klinikums rechts der Isar erhoben und anonymisiert ausgewertet. Ergebnisse. Trotz steigender Patientenzahlen von 2019 auf 2020 kam es von Februar auf März 2020 zu einem deutlichen Abfall der Notfälle bis auf ein Niveau unter das von 2019, der im April weiter anhielt. Dies betraf insbesondere den Fachbereich Unfallchirurgie mit einem Rückgang des mittleren Patientenaufkommens um etwa $40 \%$.

Im Hinblick auf die Beschwerdebilder im März 2020 zeigte sich, dass ein vermehrtes Aufkommen von Unwohlsein (+47\%) und Atemproblemen (+36\%) zu verzeichnen war, wohingegen Rückenschmerzen $(-41 \%)$, Wunden $(-29 \%)$, thorakale $(-24 \%)$ sowie abdominelle Schmerzen $(-23 \%)$ deutlich seltener vertreten waren als im Vorjahr. Bezogen auf den Schweregrad der Beschwerden wirkte sich der Rückgang vor allem auf Beschwerdebilder mit niedriger Dringlichkeitsstufe aus.

Schlussfolgerung. Im Rahmen der COVID19-Pandemie kam es zu einem deutlichen Rückgang des Patientenaufkommens in einer der größten Notaufnahmen in München. Dies sollte bei bestehenden Krankenhauskapazitäten vermieden werden, um potenziell gesundheitlichen Schäden durch eine aufgeschobene oder ausbleibende notfallmäßige Vorstellung vorzubeugen.

\section{Schlüsselwörter}

Notfall · Patientenaufkommen - Coronavirus . SARS-CoV-2 $\cdot$ Kollateralschaden

\section{Numbers of emergency room patients during the COVID-19 pandemic}

\section{Abstract}

Background. Since end of March, the health care system in Germany has been placed into a state of emergency in order to gain resources for the spreading coronavirus disease 2019 (COVID-19) pandemic. The overall goal of this study is to evaluate the number of emergency room patients at the time of the pandemic in order to draw conclusions about the influence of the COVID 19 pandemic on the number of patients in an emergency department. Materials and methods. With this descriptive epidemiologic study we collected and analyzed anonymized patient-related data of 19,357 cases presenting to the emergency department of the Klinikum rechts der Isar (Munich) from 01 February 2019 to
30 April 2019 and from 01 February 2020 to 30 April 2020.

Results. Despite an increase in the number of patients from 2019 to 2020, there was a significant drop in the number of emergencies from February to March 2020 and proceeding in April to a level below that of 2019. This was particularly observed in the field of trauma surgery, with a $40 \%$ decrease in the number of patients. With regard to the individual complaint patterns in March 2020 , it was found that an increased incidence of malaise $(+47 \%)$ and breathing problems $(+36 \%)$ was recorded, whereas back pain $(-41 \%)$, wounds $(-29 \%)$, thoracic $(-24 \%)$ and abdominal pain $(-23 \%)$ were significantly less common than in the previous year. In terms of the severity of the complaints, the decline was mainly due to complaints with a low degree of urgency.

Conclusion. In the course of the COVID-19 pandemic we observed a significant decline in the number of patients in one of the largest emergency rooms in Munich. This has to be avoided with existing hospital capacities, in order to prevent potential damage to health caused by postponed or missing emergency presentations.

Keywords

Emergency - Patient numbers - Coronavirus . SARS-CoV-2 Collateral damage
Version 25.0., IBM, Armonk, NY, USA; Microsoft Excel 2019; Microsoft Office, Redmond, WA, USA).

\section{Ergebnisse}

\section{Basischarakteristika}

In -Tab. 2 sind die Baseline-Charakteristika der Patienten im Beobachtungszeitraum Februar bis März für die Jahre 2019 und 2020 aufgeführt. Das Alter der Patienten lag im Mittel bei $48 \pm 21$ bzw. $50 \pm 21$ Jahre. Der Anteil an männlichen Patienten lag bei $52 \%$ respektive $53 \%$. Der Großteil der Notfallpatienten stellte sich selbstständig vor. Die Stufen grün und gelb waren in beiden Jahren erwartungsgemäß die am häufigsten im Rahmen der Triage gewählten Dringlichkeiten.

Bezüglich der Fachbereiche in den Jahren 2019 und 2020 hatte Chirurgie mit $65 \%$ bzw. $53 \%$ den größten Anteil, gefolgt von Konservativ mit $34 \%$ bzw. $45 \%$ und Andere mit $1 \%$ bzw. $2 \%$.

\section{Patientenzahlen gesamt}

Im Februar 2019 wurden in der zentralen Notaufnahme (ZNA) insgesamt 2941 Patienten behandelt. Im Mittel entspricht dies in etwa 105 Patientenkontakten täglich. Im Februar 2020 wurden insgesamt 3796 (davon 0 COVID-19-)Patienten versorgt, das entspricht also durchschnittlich etwa 131 Patientenkontakte pro Tag. Somit zeigt sich im Jahr 2020 im Vergleich zum Vorjahr ein Mehraufkommen von $26 \%$. 
Tab. 2 Basischarakteristika der untersuchten Fälle im Beobachtungszeitraum 2019 und 2020

\begin{tabular}{|c|c|c|c|c|}
\hline \multicolumn{2}{|l|}{ Charakteristika } & \multirow{2}{*}{$\begin{array}{l}2019(N=9795) \\
48 \pm 21\end{array}$} & \multirow{2}{*}{$\begin{array}{l}2020(N=9562) \\
50 \pm 21\end{array}$} & \multirow{2}{*}{$\begin{array}{l}\boldsymbol{p} \text {-Wert } \\
<0,001\end{array}$} \\
\hline Alter, $M W \pm S D$ & & & & \\
\hline Geschlecht, $n$ (\%) & Männlich & $5085(52 \%)$ & $5108(53 \%)$ & 0,036 \\
\hline \multirow[t]{3}{*}{ Einweisungsart, $n$ (\%) } & Selbst & 7118 (73\%) & $6144(64 \%)$ & $<0,001$ \\
\hline & Rettungsdienst & 1837 (19\%) & $2732(29 \%)$ & $<0,001$ \\
\hline & Keine Daten & $840(9 \%)$ & $686(7 \%)$ & $<0,001$ \\
\hline \multirow[t]{5}{*}{ Dringlichkeit, $n$ (\%) } & Blau & $1164(12 \%)$ & $821(9 \%)$ & $<0,001$ \\
\hline & Grün & $5426(55 \%)$ & $4618(48 \%)$ & $<0,001$ \\
\hline & Gelb & $2560(26 \%)$ & 3487 (37\%) & $<0,001$ \\
\hline & Orange & $486(5 \%)$ & $519(5 \%)$ & 0,144 \\
\hline & Rot & $159(2 \%)$ & $117(1 \%)$ & 0,019 \\
\hline \multirow[t]{3}{*}{ Fachbereich, $n$ (\%) } & Chirurgie & $6391(65 \%)$ & $5051(53 \%)$ & $<0,001$ \\
\hline & Konservativ & $3326(34 \%)$ & $4345(45 \%)$ & $<0,001$ \\
\hline & Andere & $78(1 \%)$ & $166(2 \%)$ & $<0,001$ \\
\hline \multirow[t]{3}{*}{ Entlassart, $n(\%)$} & Ambulant & $7816(80 \%)$ & $7159(75 \%)$ & $<0,001$ \\
\hline & Stationär & $1976(20 \%)$ & $2395(25 \%)$ & $<0,001$ \\
\hline & Verstorben & $3(0 \%)$ & $8(0,1 \%)$ & 0,122 \\
\hline
\end{tabular}

Im März 2019 wurden insgesamt 3333 Patienten in der ZNA behandelt (108 Patienten pro Tag). Im März des Folgejahrs waren es 3101 (davon 97 COVID-19-)Patienten (100 Patienten pro Tag). Demnach kam es im März 2020 im Vergleich zum Vorjahr sogar zu einem Abfall der Notfallpatienten um etwa 7\%.

Im April 2019 wurden in der ZNA insgesamt 3521 Patienten behandelt (117 Patienten pro Tag). Im April des Jahrs 2020 beliefen sich die Zahlen auf 2665 (davon 56 COVID-19-)Patienten (89 Patienten pro Tag), was einem Rückgang an Notfallpatienten von etwa $24 \%$ entspricht.

Die Patientenzahlen im untersuchten Zeitraum 2019 waren weitgehend konstant mit einer Abweichung von etwa 3 Patienten pro Tag und zeigten von $\mathrm{Fe}$ bruar bis April einen Anstieg von etwa 12 Patienten pro Tag. Im Jahr 2020 dagegen zeigt sich von Februar bis April ein deutlicher Abfall der Patienten um etwa 42 Patienten pro Tag, was einem Rückgang von etwa $32 \%$ pro Tag entspricht (• Abb. 1).

\section{Fachbereiche}

Im Fachbereich „Konservativ“ wurden im Zeitraum Februar bis April 2019 insgesamt 3326 Patienten behandelt, im selben Zeitraum 2020 waren es insgesamt 4345 Patienten, was einem signifikan-
März und bis April stark ab, um etwa 20 bzw. 27 Patienten pro Tag.

In der Subdisziplin Unfallchirurgie wurden im Beobachtungszeitraum 2019 insgesamt 5136 Patienten versorgt. Im gleichen Zeitraum des Jahrs 2020 waren es 3944 Patienten. Dies entspricht einem Abfall des Patientenaufkommens um etwa $23 \%$ bzw. 14 Patienten pro Tag. Umso deutlicher wird dies, wenn man die Änderung der Patientenzahlen im Monatsvergleich betrachtet (- Abb. 2b). Hier zeigt sich für das Jahr 2019 ein leichter Anstieg des Patientenaufkommens von Februar auf März um etwa 4 Patienten pro Tag, bis April um etwa 10 Patienten pro Tag. Im Jahr 2020 stellt sich stattdessen ein deutlicher Abfall von Februar auf März um etwa 16 Patienten pro Tag, bis April sogar um etwa 21 Patienten pro Tag dar.

Im Fachbereich „Andere“ wurden im beobachteten Zeitraum des Jahrs 2019 insgesamt 78 Patienten behandelt, während es im Jahr 2020 etwa 166 Patienten waren. Von Februar bis April desselben Jahrs blieben die Zahlen sowohl in 2019 als auch in 2020 weitgehend konstant mit einer maximalen Schwankung von etwa 0,3 Patienten pro Tag.

Bezogen auf die 2 wichtigen Fachbereiche „Konservativ" und „Chirurgie“ zeigt sich, dass im Jahr 2020 von Februar bis April ein zum Vorjahr überproportional deutlicher Rückgang der Patientenzahlen $\mathrm{zu}$ verzeichnen ist.

\section{Dringlichkeit}

Vergleicht man das Patientenaufkommens der Jahre 2019 und 2020 miteinander, ist klar zu erkennen, dass die Dringlichkeitsstufe "gelb“ zugewinnt, wohingegen die Patientenzahlen für „blau“ und "grün“ zurückgingen (• Abb. 3).

\section{Beschwerdebilder}

In - Tab. 3 ist die Verteilung der Notfälle anhand der 51 Beschwerdebilder gemäß MTS für den Beobachtungszeitraum 2019 und 2020 aufgeführt. Die häufigsten 3 Beschwerdebilder in den Monaten Februar bis April für die Jahre 2019 und 2020 waren demnach „Extremitätenprobleme“ (2350 vs. 2005 Pati- 


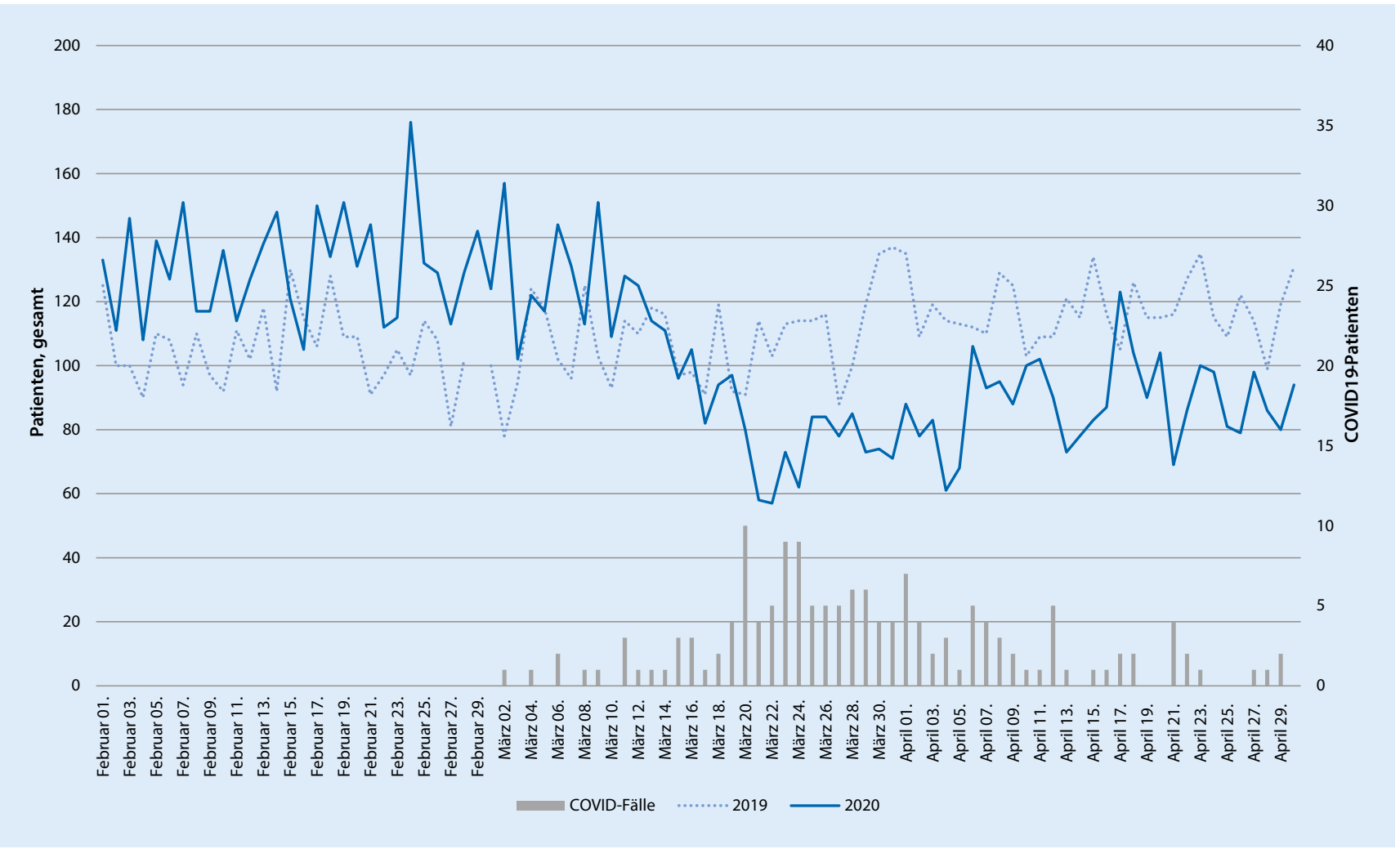

Abb. 1 ॥ Entwicklung des Patientenaufkommens und der COVID-19(Coronavirus-disease-2019)-Fälle in der zentralen Notaufnahme des Klinikums rechts der Isar von Februar bis April in den Jahren 2019 und 2020. Die gestrichelte orangefarbene Linie entspricht dem täglichen Patientenaufkommen im Jahr 2019, die blaue Linie dem im Jahr 2020. Graue Säulen entsprechen den bestätigten COVID-19-Fällen, die über die ZNA aufgenommen wurden

enten), „Unwohlsein bei Erwachsenen“ (1171 vs. 1745 Patienten) und „Abdominelle Schmerzen bei Erwachsenen“" (1019 vs. 915 Patienten).

Um die Auswirkung der COVID-19Pandemie auf die einzelnen Beschwerdebilder zu untersuchen, haben wir diese im März 2020, dem Monat mit dem höchsten Aufkommen von COVID-19-Patienten (• Abb. 1), mit dem Vorjahr verglichen (• Abb. 4). Hier zeigt sich eine deutliche Zunahme von „Unwohlsein bei Erwachsenen" (+47\% zum Vorjahr), „Atemprobleme bei Erwachsenen“ (+36\% zum Vorjahr), während „Rückenschmerz“ (-41\% zum Vorjahr), „Wunden“ (-29\% zum Vorjahr) und „Thoraxschmerz" (-24\% zum Vorjahr) im Vergleich zum Vorjahr deutlich abgenommen haben.

\section{Diskussion}

Am Klinikum rechts der Isar stellen sich jedes Jahr mehr als 40.000 Patienten in der zentralen Notaufnahme vor. Vergleicht man die Patientenzahlen im untersuchten Zeitraum der Jahre 2019 und 2020 sind die Notfälle auf vergleichbarem Niveau mit einer leicht steigenden Tendenz in 2020 (• Abb. 1). Es zeigt sich jedoch über alle Fachbereiche hinweg ein deutlicher Abfall an Notfallpatienten von Februar bis April 2020. Das tägliche Patientenaufkommen von 131 Patienten pro Tag im Februar fällt ab dem 10. März auf etwa 88 Patienten, also um etwa $32 \%$, für den restlichen Monat und hält bis einschließlich April an (• Abb. 1). Zeitgleich mit den sinkenden Zahlen an Notfallpatienten stiegen die bestätigten COVID19-Fälle im Münchener Raum und auch am Klinikum rechts der Isar. Ab dem 20. März werden über die ZNA täglich mindestens 4 bestätigte COVID-19-Fälle aufgenommen (• Abb. 1).

Für den starken Einbruch an Notfallpatienten in der 2. Märzhälfte müssen mehrere Faktoren in Betracht gezogen werden. Hauptursächlich scheint je- doch der spürbare Beginn der COVID19-Pandemie in Deutschland zu sein.

Betrachtet man die Fachbereiche getrennt voneinander, ist $\mathrm{zu}$ erkennen, dass die Anzahl der Notfallpatienten mit unfallchirurgischen Krankheitsbildern im Vergleich zu den konservativen Notfallpatienten mit Erkrankungen aus anderen Fachbereichen deutlich abgefallen ist (•Abb. 2). Ursächlich hierfür könnte unter anderem die in Bayern ab dem 21.03.2020 verfügte Ausgangssperre sein [1]. Die Mobilität und sportlichen Aktivitäten sind hierdurch für die Bevölkerung deutlich eingeschränkt worden. Alle öffentlichen Spielplätze und Sportstätten, wie Fitnessstudios, Freizeitbäder, etc., wurden geschlossen und Mannschaftssportarten, wie Fußball, Basketball, etc., durften nicht mehr stattfinden. Ein Rückgang der Mobilität und sportlichen Aktivität könnte somit zu weniger Fällen mit unfallchirurgischen Krankheitsbildern führen. 


\section{Originalien}

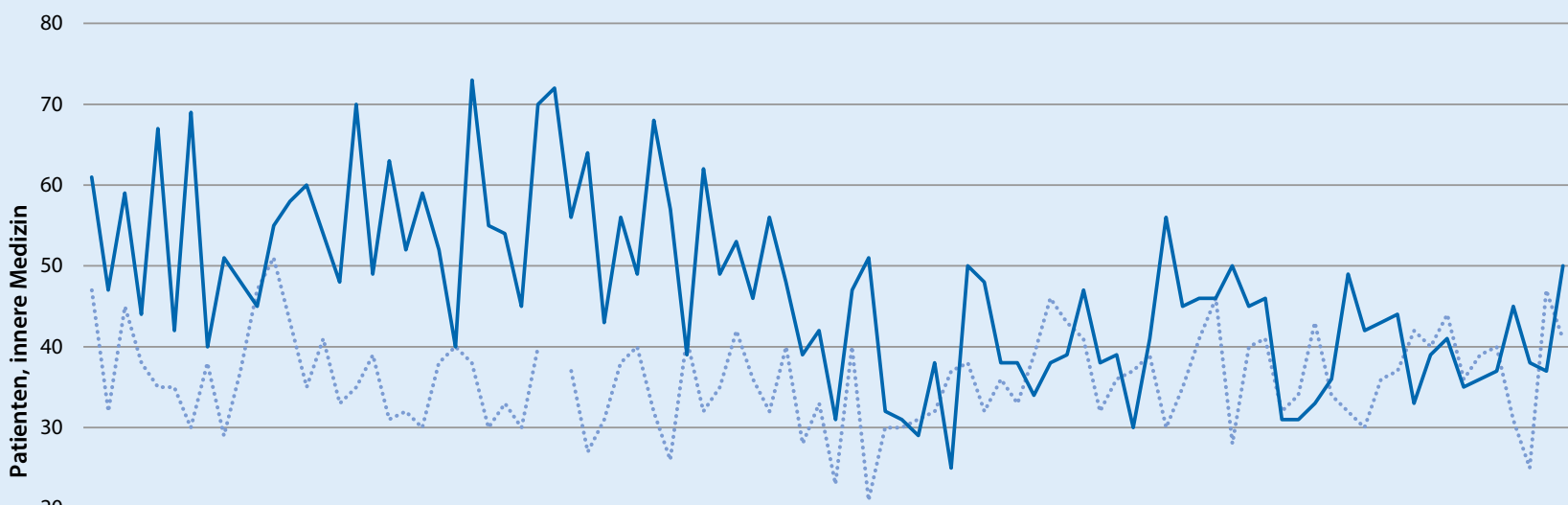

10

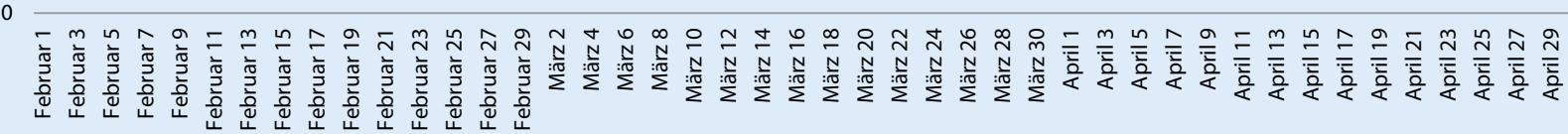

a

$2019-2020$

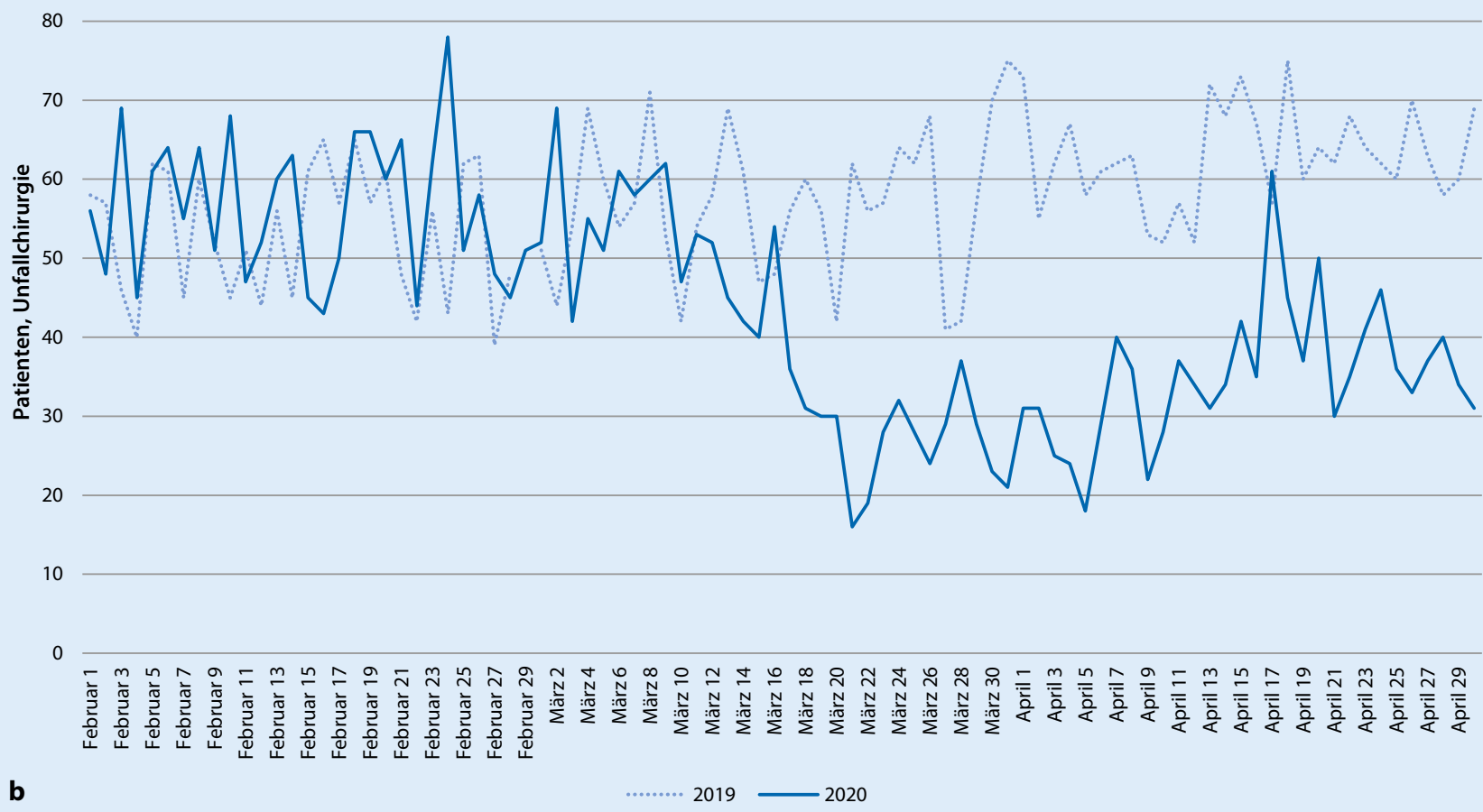

Abb. $2 \Delta$ Entwicklung der Patientenzahlen für den Fachbereich innere Medizin (a) und den Fachbereich Unfallchirurgie (b) von Februar bis A pril in den Jahren 2019 und 2020. Die gestrichelte Linie entspricht dem täglichen Patientenaufkommen in 2019, die durchgezogene Linie dem in 2020 


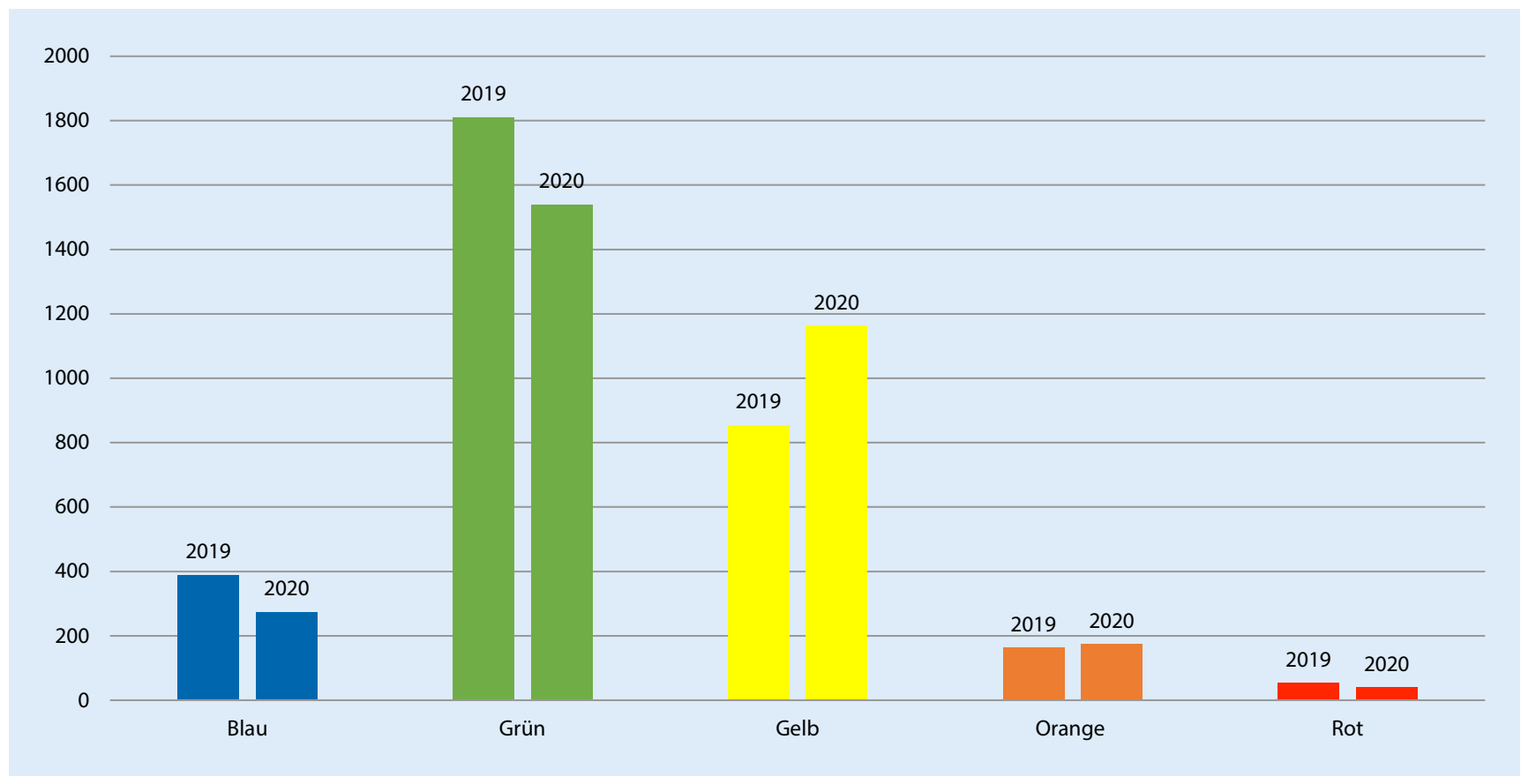

Abb. 3 A Direkter Vergleich des Patientenaufkommens für die Jahre 2019 und 2020 gentrennt nach den Dringlichkeitsstufen nach MTS (Manchester Triage System)

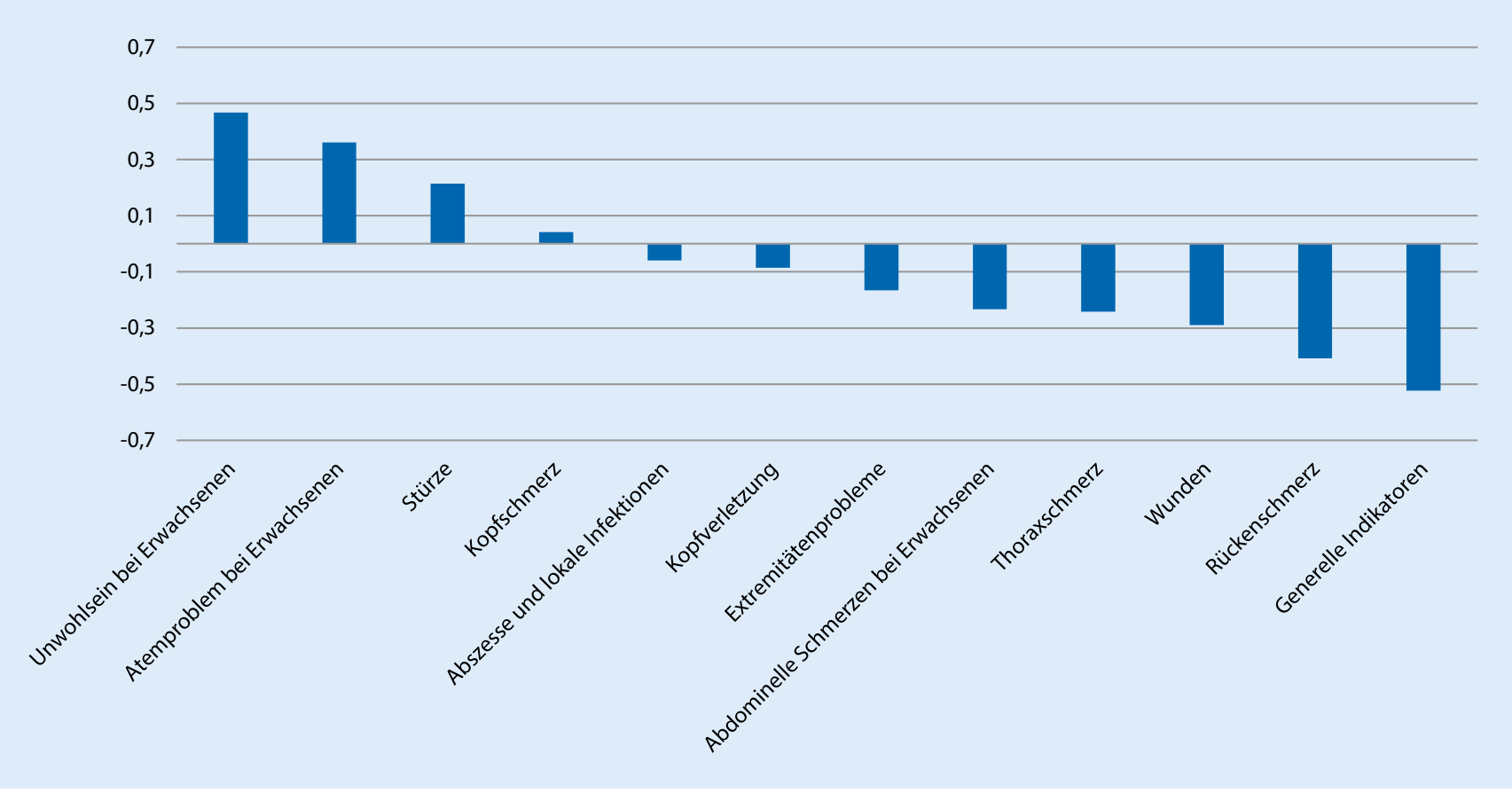

Abb. 4 A Relative Veränderung der Fallzahl pro Beschwerdebild nach MTS ( $N \geq 50$ pro Monat) im März 2020 im Vergleich zum Vorjahr in Prozent

Bestätigt wird dies durch eine deutliche Umverteilung der Beschwerdebilder, die im Rahmen der Triage erhoben werden. Wie in Abb. 4 zu erkennen haben im Vergleich zum Jahr 2019 im März 2020 beispielsweise Atemwegs- probleme deutlich zugenommen und demgegenüber Kopfverletzungen und Rückenschmerzen deutlich abgenommen. Derartige Schwankungen können ebenfalls saisonal bedingt sein, insbesondere durch die jährlich auftretende
Influenza- und Respiratory-SinzytialVirus(RSV)-Welle. Diese fiel im Jahr $2020 \mathrm{im}$ Vergleich zu den vergangenen 5 Jahren mit einer Dauer von 11 Wochen allerdings deutlich kürzer aus und endete am 22.03.2020 [13]. Ebenso sind 


\section{Originalien}

Tab. 3 Verteilung der Patientenzahlen auf die einzelnen Beschwerdebilder je Monat

Beschwerdebild

2019

2020

Abdominelle Schmerzen bei Erwachsenen

Februar

Februar

Abdominelle Schmerzen bei Kindern

287

360

372

381

März

April

Abszesse und lokale Infektionen

0

Allergie

Angriff (Zustand nach)

Asthma

Atemproblem bei Erwachsenen

60

4

2

1

85

23

24

0

Atemproblem bei Kindern

\section{0}

Auffälliges Verhalten

0

Augenprobleme

Besorgte Eltern

Betrunkener Eindruck

Bisse und Stiche

Chemikalienkontakt

Diabetes

Durchfälle und Erbrechen

Extremitätenprobleme

Fremdkörper

Gastrointestinale Blutung

Generelle Indikatoren

Gesichtsprobleme

Halsschmerzen

Hautausschläge

Herzklopfen

Hodenschmerz

Körperstammverletzung

Kollaps

Kopfschmerz

Kopfverletzung

Krampfanfall

Misshandeltes Kind

Nackenschmerz

Ohrenprobleme

Psychiatrische Erkrankung

Rückenschmerz

Reanimation

Schreiendes Baby

Schwangerschaftsproblem

Schweres Trauma

Selbstverletzung

Sexualinfektion

Stürze

Thoraxschmerz

Überdosierung und Vergiftung

Unwohlsein bei Erwachsenen

Unwohlsein bei Kindern

Urologische Probleme 


\begin{tabular}{|c|c|c|c|c|c|c|}
\hline \multirow[t]{2}{*}{ Beschwerdebild } & \multicolumn{3}{|l|}{2019} & \multicolumn{3}{|l|}{2020} \\
\hline & Februar & März & April & Februar & März & April \\
\hline Vaginale Blutung & 2 & 1 & 3 & 7 & 3 & 3 \\
\hline Verbrennungen und Verbrühungen & 12 & 17 & 1 & 8 & 3 & 5 \\
\hline Wunden & 125 & 190 & 166 & 168 & 135 & 153 \\
\hline Zahnprobleme & 4 & 1 & 4 & 10 & 6 & 8 \\
\hline Gesamtergebnis & 2941 & 3333 & 3521 & 3796 & 3101 & 2665 \\
\hline
\end{tabular}

die Todeszahlen im Jahr 2020 mit 518 influenzabedingten Todesfällen um etwa $54 \%$ niedriger als im Vorjahr $[13,14]$.

Ein weiterer Grund für den Rückgang an Notfallpatienten könnte die sinkende Zahl an Selbsteinweisungen sein. Infolge der medialen Präsenz der COVID19-Pandemie und des erhöhten Ansteckungsrisikos in Krankenhäusern wäre es denkbar, dass sich derartige Bedenken auf die Konsultationsfrequenz der Patienten auswirken. Die Bekanntgabe der ersten Todesfälle in Deutschland am 09. März könnte ebenfalls dazu beigetragen haben [12]. Denkbar wäre ebenso, dass Beschwerden hierdurch länger ausgehalten werden bzw. ganz auf einen Arztbesuch verzichtet wird.

Auch durch unterschiedliche Empfehlungen einzelner Fachgesellschaften, des RKI, der WHO, der Bundesländer und der EU-Staaten, herrscht unter der Bevölkerung Unsicherheit über den richtigen Umgang mit Schutzmaßnahmen im Rahmen der Pandemie. Durch fehlende soziale Kontakte könnten auch Faktoren wegfallen wie z. B. ein freundschaftlicher Rat, sich in ärztliche Behandlung zu begeben. Bei Fehlen dieser laienbasierten Unterstützung wird so der Gang ins Krankenhaus verzögert.

Auch die veränderte Verteilung der Dringlichkeit von „blau“ und „grün“ hin zu „gelb“, also zu einer zeitkritischeren Vorstellung, sowie die signifikante $\mathrm{Zu}$ nahme der stationären Aufnahmen um $5 \%$ im Jahr 2020 im Vergleich zum Vorjahr sprechen ebenfalls dafür, dass Patienten mit einer vermutlichen Bagatelle eher zu Hause bleiben und sich eher solche mit schwerwiegenderen Krankheitsbildern vorstellen. Es bleibt allerdings ungewiss, ob Patienten möglicherweise aufgrund dieser Bedenken so lange gewartet haben, bis eine ernstzunehmen- de Erkrankung erwachsen ist. Daten, die diese Vermutung erhärten könnten, wie z. B. die Verweildauer oder Dauer der ggf. nötigen intensivmedizinischen Behandlung, liegen leider nicht vor.

Ein sinnvoller Ansatz, um derartige Kollateralschäden, die möglicherweise durch einen verzögerten oder ausgesetzten Krankenhausbesuch entstehen, zu vermeiden, wäre, der Bevölkerung Ängste zu nehmen, die Kommunikation über Schutzausrüstung und Ansteckungsgefahr im Krankenhaus zu verbessern und auf die gute Infrastruktur des deutschen Gesundheitssystem zu verweisen. Denn trotz eines hohen Aufkommens an COVID-19-Patienten bestanden zu jedem Zeitpunkt ausreichende Kapazitäten, um alle Notfälle adäquat behandeln zu können.

\section{Limitationen}

Im Rahmen der Auswertung des IT-Systems ERPath wurden keine weiterführenden Basisdaten zu den Patienten erhoben, daher kann auch keine Aussage zur tatsächlichen Krankheitsschwere der einzelnen Notfälle gemacht werden. Die Auswertung gibt auch keine Information über den weiteren Verlauf der Patienten. Daten zur Entlassart oder zum stationären Verlauf liegen nicht vor. Ebenso haben wir keinen Zugriff auf Daten aus dem ambulanten Sektor, die Aufschluss über die Auslastung der Hausarztpraxen geben könnten.

Warum die Patientenzahlen mit Beginn der Behandlung der ersten COVID19-Fälle gesunken sind, kann nicht mit letzter Sicherheit geklärt werden.

Weiterführende Daten wie ICD-10Hauptdiagnose oder stationäre Behandlungsdauer wären zur weiteren Bestim- mung von möglichen Kollateralschäden von erheblicher Bedeutung.

\section{Fazit für die Praxis}

- Das Patientenaufkommen in der Notaufnahme ist zur Zeit der COVID-19(Coronavirus-disease-2019)Pandemie gesunken.

- Ursächlich könnten unseres Erachtens auch unbegründete Ängste in der Bevölkerung über eine mögliche Infektion im Krankenhaus sein.

- Bessere Kommunikation auf allen Kanälen könnte den Patienten die Sicherheit geben, sich im Notfall in ein Krankenhaus zu begeben.

- Bei bestehenden Krankenhauskapazitäten müssen Kollateralschäden der COVID-19-Pandemie vermieden werden.

\section{Korrespondenzadresse}

\section{Dr. M. Dommasch}

Zentrale interdisziplinäre Notaufnahme, Klinikum rechts der Isar, Technische Universität München

Ismaninger Str. 22, 81675 München, Deutschland

michael.dommasch@mri.tum.de

Funding. Open Access funding provided by Projekt DEAL.

\section{Einhaltung ethischer Richtlinien}

Interessenkonflikt. T. Tschaikowsky, A. Becker von Rose, S. Consalvo, P. Pflüger, P. Barthel, C.D. Spinner, B. Knier, K.-G. Kanz und M. Dommasch geben an, dass kein Interessenkonflikt besteht.

Für diesen Beitrag wurden von den Autoren keine Studien an Menschen oder Tieren durchgeführt. Für die aufgeführten Studien gelten die jeweils dort angegebenen ethischen Richtlinien. 
Open Access. Dieser Artikel wird unter der Creative Commons Namensnennung 4.0 International Lizenz veröffentlicht, welche die Nutzung, Vervielfältigung, Bearbeitung, Verbreitung und Wiedergabe in jeglichem Medium und Format erlaubt, sofern Sie den/die ursprünglichen Autor(en) und die Quelle ordnungsgemäß nennen, einen Link zur Creative Commons Lizenz beifügen und angeben, ob Änderungen vorgenommen wurden.

Die in diesem Artikel enthaltenen Bilder und sonstiges Drittmaterial unterliegen ebenfalls der genannten Creative Commons Lizenz, sofern sich aus der Abbildungslegende nichts anderes ergibt. Sofern das betreffende Material nicht unter der genannten Creative Commons Lizenz steht und die betreffende Handlung nicht nach gesetzlichen Vorschriften erlaubt ist, ist für die oben aufgeführten Weiterverwendungen des Materials die Einwilligung des jeweiligen Rechteinhabers einzuholen.

Weitere Details zur Lizenz entnehmen Sie bitte der Lizenzinformation auf http://creativecommons.org/ licenses/by/4.0/deed.de.

\section{Literatur}

1. Bayerisches Staatsministerium für Gesundheit und Pflege (2020) Bekanntmachung vom 20.03.2020, Vollzug des Infektionsschutzgesetzes (IfSG) Vorläufige Ausgangsbeschränkung anlässlich der Corona-Pandemie, Az. Z6a-G8000-2020/12298.https://www.bayern.de/wp-content/uploads/ 2020/03/20-03-20-ausgangsbeschraenkungbayern-.pdf.Zugegriffen: 10. Juni 2020

2. Bayerisches Staatsministerium für Gesundheit und Pflege (2020) Entwurf eines Gesetzes zum Ausgleich COVID-19 bedingter finanzieller Belastungen der Krankenhäuser und weiterer Gesundheitseinrichtungen vom 23.03.2020. https://www.bundesgesundheitsministerium. de/fileadmin/Dateien/3 Downloads/Gesetze und_Verordnungen/GuV/C/Entwurf_COVID-19Krankenhausentlastungsgesetz.pdf. Zugegriffen: 10. Juni 2020

3. Bayerische Staatsministerium für Gesundheit und Pflege (2020) Pressemitteilung vom 27.01.2020, Bestätigter CoronavirusFall in Bayern. https://www.stmgp.bayern. de/presse/bestaetigter-coronavirus-fall-inbayern-infektionsschutzmassnahmen-laufen/? output=pdf. Zugegriffen: 10 . Juni 2020

4. Bayerisches Staatsministerium des Innern, für Sport und Integration Pressemitteilung vom 16.03.2020, Hermann stellt Katastrophenfall fest. https://www.stmi.bayern.de/med/ pressemitteilungen/pressearchiv/2020/96/index. php. Zugegriffen: 10. Juni 2020

5. Bayerischen Staatsministeriums des Innern, für Sport und Integration, Bayerischen Staatsministeriums für Gesundheit und Pflege (2020) Bekanntmachung vom 24. März 2020: Notfallplan Corona-Pandemie: Allgemeinverfügung zur Bewältigung erheblicher Patientenzahlen in Krankenhäusern. https://www. stmgp.bayern.de/wp-content/uploads/2020/ 03/allgemeinverfuegung-notfallplan-coronaentwurf-final-stand-24-03-1443-uhr-002.pdf. Zugegriffen: 10. Juni 2020

6. Bundesregierung (2020) Bundesregierung, Bekanntmachung vom 12.03.2020: Sozialkontakte vermeiden, Ausbreitung verlangsamen. https:// www.bundesregierung.de/breg-de/themen/ coronavirus/mpk-1730186. Zugegriffen: 10. Juni 2020

7. Deutsches Ärzteblatt (2020) Robert-Koch-Institut stuft Risiko für die Bevölkerung jetzt als „hoch“ ein. https://www.aerzteblatt.de/nachrichten/ 111108/Robert-Koch-Institut-stuft-Risiko-fuerdie-Bevoelkerung-jetzt-als-hoch-ein. Zugegriffen: 10 . Juni 2020

8. ERPath (2020) Das Notaufnahmen-Informationssystem. https://www.ehealth-tec.de/services/ erpath-notaufnahmeninformationssystem. Zugegriffen: 10. Juni 2020

9. Gemeinsamer Bundesausschuss (2018) Regelungen zu einem gestuften System von Notfallstrukturen in Krankenhäusern gemäß § 136c Absatz 4 des Fünften Buches Sozialgesetzbuch (SGB V). https://www.g-ba.de/downloads/62-4921598/Not-Kra-R_2018-04-19_iK2018-05-19.pdf. Zugegriffen: 10. Juni 2020

10. Mackway-Jones K, Marsden J, Windle J (Hrsg) (2011) Ersteinschätzung in der Notaufnahme: Das Manchester-Triage-System Bd. 3. Huber, Bern

11. Portal München Betriebs-GmbH (2020) CoronaLockerungen: Ausgangsbeschränkung entfällt zum 6.5. In: muenchen.de. 5. Mai 2020. https:// www.muenchen.de/aktuell/2020-05/coronalockerungen-ausgangsbeschraenkung-wird-zurkontaktbeschraenkung.html.Zugegriffen: 10. Juni 2020

12. Robert Koch-Institut (2020) Archiv der Situationsberichte zu COVID-19, Situationsbericht 09.03.2020. https://www.rki.de/DE/ Content/InfAZ/N/Neuartiges_Coronavirus/ Situationsberichte/2020-03-09-de.pdf? blob=publicationFile. Zugegriffen: 10. Juni 2020

13. Robert Koch-Institut (2019) Bericht zur Epidemiologie der Influenza in Deutschland Saison 2018/19. https://influenza.rki.de/Saisonberichte/2018.pdf. Zugegriffen: 10. Juni 2020

14. Robert Koch-Institut (2020) Influenza-Wochenbericht, Kalenderwoche 20/2020 (09.05 bis 15.05.2020). https://influenza.rki.de/ Wochenberichte/2019_2020/2020-20.pdf. Zugegriffen: 10. Juni 2020

15. Shi H, Han X, Jiang N et al (2020) Radiological findings from 81 patients with COVID-19 pneumonia in Wuhan, China: a descriptive study. Lancet Infect Dis 20:425-434

16. Weltgesundheitsorganisation (2020) Weltgesundheitsorganisation (WHO) erklärt COVID19-Ausbruch zur Pandemie. http://www.euro. who.int/de/health-topics/health-emergencies/ coronavirus-covid-19/news/news/2020/3/whoannounces-covid-19-outbreak-a-pandemic. Zugegriffen: 10 . Juni 2020

17. Weltgesundheitsorganisation (2020) Weltgesundheitsorganisation (WHO), Erklärung des Generaldirektors der WHO über die Tagung des IGV-Notfallausschusses zum Neuartigen Coronavirus (2019-nCoV). https://www.who. int/dg/speeches/detail/who-director-generals-statement-on-ihr-emergency-committee-onnovel-coronavirus-(2019-ncov). Zugegriffen: 10 Juni 2020

18. Zhang J,Zhou L, Yang Yetal (2020) Therapeuticand triage strategiesfor 2019 novel coronavirus disease in fever clinics. Lancet Respir Med 8:e11-e12
Springer Medizin Podcast

Medizin für Gesundheitsprofis

Der Podcast von SpringerMedizin.de geht seit Juli 2020 spannenden Fragen aus der Welt der Medizin nach immer freitags erweitert eine neue Folge das bereits bestehende OnlineAngebot.

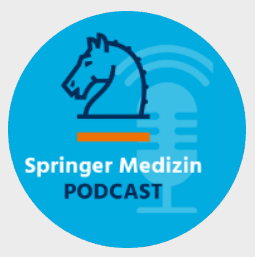

„Mit unserem

Podcast möch-

ten wir all jene ansprechen, die sich auf medizinische Themen in einer, gewissen

Flughöhe' einlassen möchten", erklärt Dr. Erik Heintz, Chefredakteur von SpringerMedizin.de das neue Format. „Gemeint sind damit Menschen, die sich nicht mit medizinischem Halbwissen zufrieden geben und gerne mehr wissen möchten. Daher legen unsere Redakteurinnen und Redakteure in München und Heidelberg vor allem auf den inhaltlichen Anspruch großen Wert. Unter Bezugnahme auf neue Studien, neue Erkenntnisse und praxisrelevantes Wissen bereiten wir jeden Podcast gründlich vor. Die Themen drehen sich um Gesundheit, Krankheit, Diagnostik, Therapie und Prävention, zu denen wir unsere Fachexpertinnen und Fachexperten aus verschiedenen medizinischen Fachgebieten hinzuziehen."

So entstehen abwechslungsreiche Folgen, die sowohl detailliert Leitlinien abarbeiten, der Evidenz von neuen Studienergebnissen auf den Grund gehen, aber auch die Hintergründe zu Erkrankungen und Therapien aufschlüsseln - sodass einerseits die ärztliche Praxis erleichtert und konkrete Tipps für die alltägliche Arbeit vermittelt werden, andererseits aber auch der Horizont über das eigene Fachgebiet hinaus erweitert werden kann.

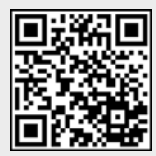

www.springermedizin.de/podcast 